\title{
Modifications in the Si valence band after ion-beam-induced oxidation
}

\author{
J. L. Alay \\ IMEC, Kapeldreef 75, B-3001 Leuven, Belgium and LCMM, Universitat de Barcelona, Diagonal 645, \\ E-08028 Barcelona, Catalonia, Spain \\ W. Vandervorst \\ IMEC, Kapeldreef 75, B-3001 Leuven, Belgium
}

(Reccived 4 October 1993; accepted 21 March 1994)

\begin{abstract}
The changes undergone by the Si surface after oxygen bombardment have special interest for acquiring a good understanding of the $\mathrm{Si}^{+}$-ion emission during secondary ion mass spectrometry (SIMS) analysis. For this reason a detailed investigation on the stoichiometry of the builtup surface oxides has been carried out using in situ X-ray photoemission spectroscopy (XPS). The XPS analysis of the Si $2 p$ core level indicates a strong presence of stiboxide chemical states when bombarding at angles of incidence larger than $30^{\circ}$. In this work a special emphasis on the analysis and interpretation of the valence band region was made. Since the surface stoichiometry or degree of oxidation varies with the angle of incidence, the respective valence band structures also differ. A comparison with experimentally measured and theoretically derived $\mathrm{Si}$ valence band and $\mathrm{SiO}_{2}$ valence band suggests that the new valence bands are formed by a combination of these two. This arises from the fact that $\mathrm{Si}-\mathrm{Si}$ bonds are present on the Si-suboxide molecules, and therefore the corresponding $3 p-3 p$ Si-like subband, which extends towards the Si Fermi level, forms the top of the respective new valence bands. Small variations in intensity and energy position for this subband have drastic implications on the intensity of the $\mathrm{Si}^{+}$-ion emission during sputtering in SIMS measurements. A model combining chemically enhanced emission and resonant tunneling effects is suggested for the variations observed in ion emission during $\mathrm{O}_{2}^{+}$bombardment for Si targets.
\end{abstract}

\section{INTRODUCTION}

The use of reactive ions $\left(\mathrm{O}_{2}^{+}\right.$and $\left.\mathrm{Cs}^{+}\right)$during secondary ion mass spectrometry (SIMS) profiling has become standard practice since it provides high ionization efficiency. It has been observed that in the case of $\mathrm{O}_{2}^{+}$bombardment of $\mathrm{Si}$ the substrate becomes partially or completely oxidized, depending on the experimental conditions. This oxidation not only has an effect on the ionization yield but also plays a substantial role during depth profiling when redistribution phenomena such as impurity segregation are active. ${ }^{1,2}$

Although there exist a large range of experimentai conditions whereby one can correlate the variation in secondary ion emission with the increasing degree of oxidation, it is also observed that under conditions of nearly complete oxidation $\left(>90 \% \mathrm{SiO}_{2}\right.$ ) still drastic changes in secondary ion emission can be seen. The origin of these changes has not yet been clarified.

Different authors have proposed some ionization mechanisms during sputtering. The most important are the electron tunneling mode ${ }^{3-6}$ and the local bond breaking model. ${ }^{6-11}$ Both models intend to explain the electronic changes undergone by an atom sputtered by ion bombardment of the target surface. An accurate description of these changes would provide us with a better quantification of ion yields in SIMS analyses. In the tunneling model, which has been tested in metals, electrons tunnel between outgoing atoms and the target surface, and hence neutralization of the outgoing ion can occur. On the other hand, the local bond breaking model is based on the chemical enhancement of secondary ion emission as observed during oxygen bombardment and is applied to both ionic and partially ionic materials (oxides).
For the specific case of $\mathrm{Si}^{+}$emission from ion-beamoxidized Si surfaces, a global model has not yet been developed. Since ion emission depends on the surface electronic properties of the bombarded material, it is necessary to study in detail the changes undergone by the Si valence band after oxygen bombardment. Only if the electronic properties of these materials are better understood it is then possible to formulate a general model for the ionization of outgoing atoms.

\section{EXPERIMENT}

The $\operatorname{Si}(100)$ samples were bombarded with a $5 \mathrm{keV}$ $\mathrm{O}_{2}^{+}$-ion beam at angles of incidence of $0^{\circ}, 15^{\circ}, 25^{\circ}, 30^{\circ}$, and $35^{\circ}$, with respect to the target normal, while rastering over $4 \times 4 \mathrm{~mm}^{2}$. The high-resolution in situ $\mathrm{x}$-ray photoemission spectroscopy (XPS) measurements are performed with an SSX-100 instrument and the base pressure of the analysis chamber is in the $10^{-10}$ Torr range and rises to $10^{-8}$ Torr during bombardment.

The oxidation states for silicon were derived from the $\mathrm{Si}$ $2 p$ core level by means of a detailed peak fitting. The constraints used in the peak fitting were those proposed in Refs. $12-14$.

\section{RESULTS AND DISCUSSION}

The validity of local bond breaking and tunneling models requires a detailed investigation of the changes undergone by the valence band after bombarding the targets. The investigation necessarily starts from an overview of the valence bands for pure $\mathrm{Si}$ and $\mathrm{SiO}_{2}$ surfaces. The following para- 


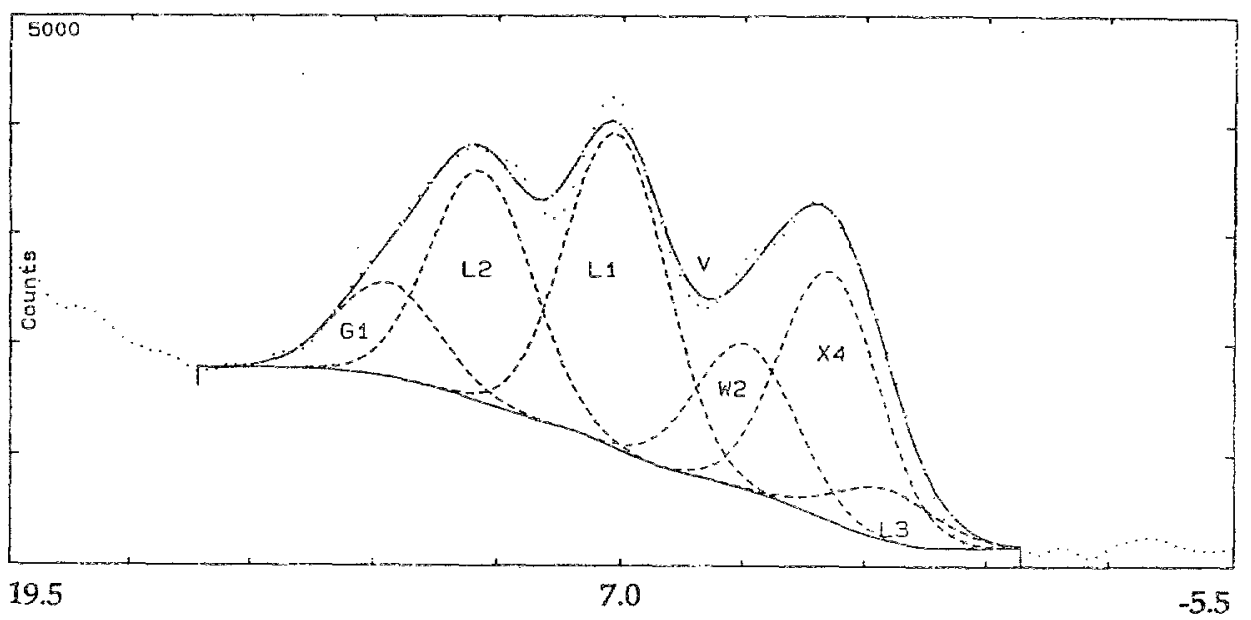

Binding Energy (eV)

FiG. 1. XPS spectrum for the valence band of $\mathrm{Si}(\mathrm{G})$ stands for $\Gamma_{1}$ ).

graphs describe the XPS valence band structure for both materials and link the results to the theoretical models which describe this energy region.

The XPS valence band for Si has been obtained from a (100) Si surface which was HF $(0.5 \%)$ dipped for 5 min and immediately loaded in the XPS chamber for analysis. The agreement between experiment and calculations is striking and allows an accurate assignment of each of the peaks [region I: $L_{3}, X_{4}$, and $\left(W_{2}+\Sigma_{1 \text { min }}\right)$; region $\mathrm{II}: L_{1}$ and $W_{1}$; region III: $L_{2}$ and $\Gamma_{1}$, and the valley between region $I$ and II (V), which can be seen in Fig. 1. Feature $W_{2}$ cannot be experimentally separated from $\Sigma_{1 \text { min }}$, although the former is predicted by empirical pseudopotential method and selfconsistent orthogonalized plane wave function calculations and the latter only by the calculations in Refs. 16 .
The $\mathrm{SiO}_{2}$ valence band has been measured on a $\mathrm{SiO}_{2}$ layer of $800 \mathrm{~mm}$, thermally grown on a (100) Si substrate. The XPS analysis was performed following the same procedures as described above for the case of crystalline Si. Theoretical caiculations ${ }^{28 \cdots 40}$ describe the upper valence bands as a group of several peaks distributed in two well-defined regions arising from very different orbital interactions between atoms: region $I$ at the top of the valence band side and region $I$ at the "strongest bond" energy side. In Fig. 2, we show the corresponding XPS spectrum. Region II accounts for the $\mathrm{Si}-\mathrm{O}$ bond occurring in a $\mathrm{O}-\mathrm{Si}-\mathrm{O}$ chain bent to an angle of about $150^{\circ}$ for amorphous $\mathrm{SiO}_{2}$. It is formed by two strong peaks and a very weak additional peak. The strong peaks correspond to $5 a_{1}$ and $4 t_{2}$ molecular orbitals arising from $\sigma$ binding between the $\mathrm{O} 2 p$ state and the Si $3 s$ and $\mathrm{Si} 3 p$

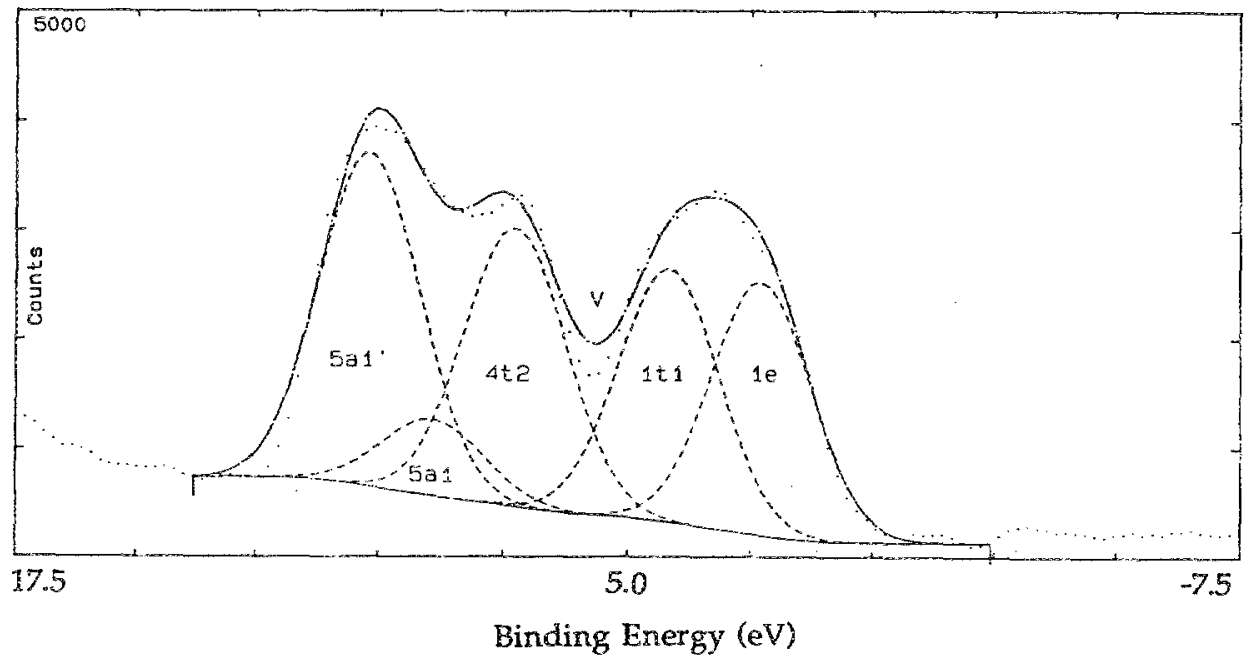

FIG. 2. XPS spectrum for the valence band of thermatly grown $\mathrm{SiO}_{2}$. 


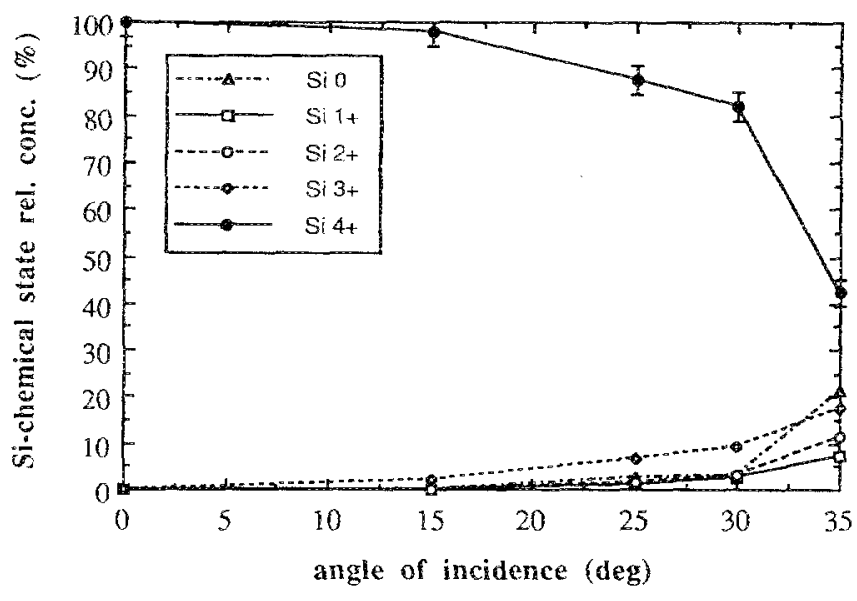

FIG. 3. Si chemical state relative concentration as a function of the angle of incidence derived from the $\mathrm{Si} 2 p$ core level fitting after $5 \mathrm{keV} \mathrm{O} \mathrm{O}_{2}^{+}$bombardment.

orbitals, respectively. Beside these two peaks we can resolve a third weak peak at the edge of the region. This peak is not assigned in the calculations of Tossel, Vaughan, and Johnson ${ }^{39}$ and we have called it $5 a_{1}^{\prime}$ (as a substructure of the $5 a_{1}$ molecular orbital). Region I is formed by the $1 e, 5 t_{2}$, and $1 t_{1}$ molecular orbitals, although the first two peaks are not resolved in the XPS spectra and form a single peak. The $5 t_{2}, 1 e$ and $1 t_{1}$ orbitals arise from nonbonding $02 p$ orbitals. Finally a rather deep valley is also measured between region $I$ and II (V).

Once the valence band for $\mathrm{Si}$ and $\mathrm{SiO}_{2}$ has been characterized in detail, we can investigate the valence bands for the surface of the altered layers, induced by ion-beam oxidation. Immediately after the steady state was reached, an XPS analysis was performed on the ion-beam-induced samples with a take-off angle of $35^{\circ}$ in order to maximize the signal from the surface. The evolution of the different Si chemical states derived from XPS data can be seen in Fig. 3. This data is based on the Si $2 p$ core level measured for different angles of incidence for the ion beam.

The valence band for the altered layers experiences a strong increase in total width from $11 \mathrm{eV}$ (crystalline Si) or $10 \mathrm{eV}\left(\mathrm{SiO}_{2}\right)$ to $15 \mathrm{eV}$. This effect arises primarily from the merging of the valence bands of $\mathrm{Si}$ and $\mathrm{SiO}_{2}$ to one single wider band for these alkered layers. The new altered valence band can be divided in two different regions. Peaks I, II, and III form the Si-like subband, while the IV, V, VI, VII, and VIII peaks form the $\mathrm{SiO}_{2}$-like valence band (Fig. 4 shows the spectrum corresponding to the valence band for an altered layer bombarded at $35^{\circ}$ ). Peaks I, II, and III correspond solely to the $L_{3}, X_{4}$, and $W_{2}+\Sigma_{1 \min }$ bands that were described as the first region in the crystalline Si valence band and they are understood as Si $3 p$-like orbitals corresponding to the presence of $\mathrm{Si}-\mathrm{Si}$ bonds in a highly damaged $\mathrm{Si}$ matrix. Peaks IV and $\mathrm{V}$ account for nonbonding $\mathrm{O} 2 p$-like molecular orbitals, as already found in the case of $\mathrm{SiO}_{2}$ $\left(1 e+5 t_{2}\right.$ and $\left.1 t_{1}\right)$, with a small influence of the $L_{1}$ and $L_{2}$ peaks ( $s-p$ hybrids) that are present in the Si valence band at this energy position. The deep valley $V$ determined for $\mathrm{SiO}_{2}$ is now partially overlapped by the $L_{2}$ peak arising from $\mathrm{Si}-\mathrm{Si}$ interactions. Peaks VI, VII, and VIII correspond to oxide bonding orbitals between $\mathrm{Si}$ and $\mathrm{O}$ and only peak $\mathrm{VI}$ becomes affected by the presence of the $\Gamma_{1}$ peak ( $s$-like orbitals) of the Si-Si interaction origin.

The zero energy for all the valence bands has been defined at the top of the valence band of $\mathrm{SiO}_{2}$. The three peaks corresponding to $\mathrm{Si}-\mathrm{Si}$ (I, II, and III) maintain the same energy position as compared to $\mathrm{Si}$, whereby the $\mathrm{Si}-\mathrm{Si} 3 p$ like orbitals remain unchanged after ion-beam-induced oxidation. The relative variations of these Si valence band features like peaks I, and II, and III should be understood as a measure of the induced suboxidation in the altered layer,

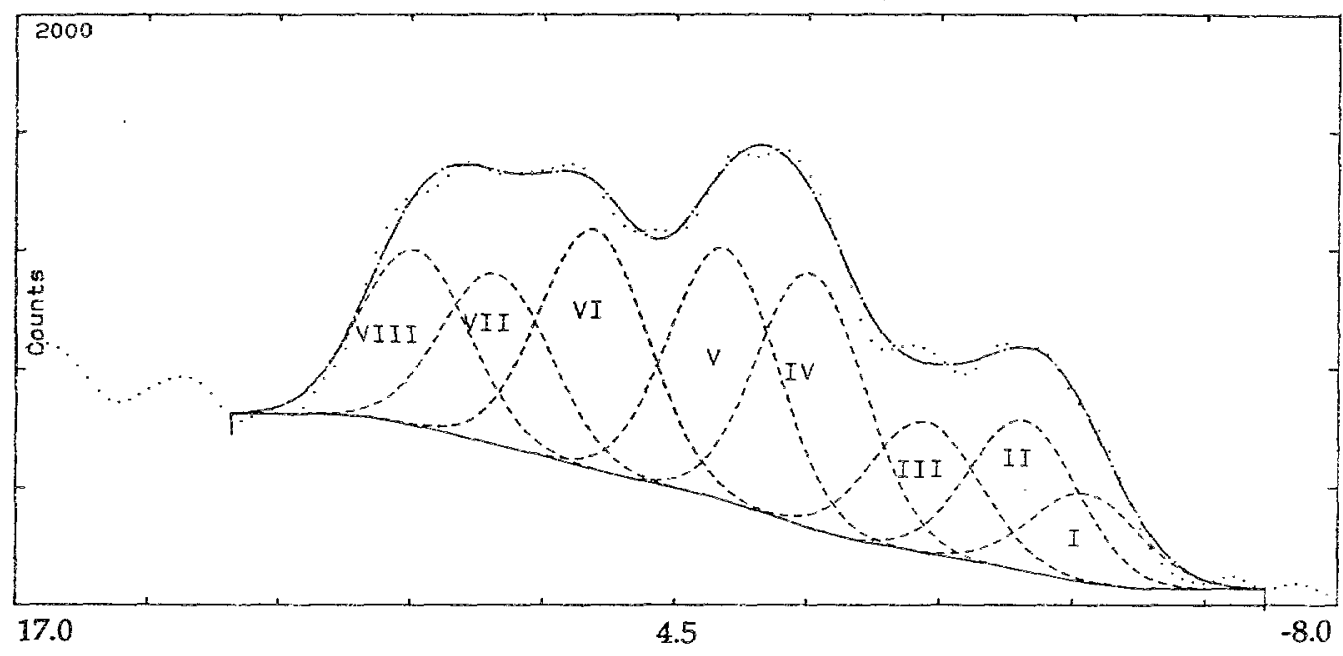

Binding Energy (eV)

Fig. 4. XPS spectrum for the valence band of the altered layer formed after a $5 \mathrm{keV} / 35^{\circ} \mathrm{O}_{2}^{+}$bomburdment of $\mathrm{Si}$. 
since the suboxide molecules include one silicon atom bond to one $\left(\mathrm{Si}^{1+}\right)$, two $\left(\mathrm{Si}^{2+}\right)$, and three $\left(\mathrm{Si}^{3+}\right)$ oxygen atoms and three, two and one silicon atoms (Si $3 p$-like orbitals) respectively. The intensity off peaks $I, I$, and III decreases with decreasing angle of incidence or, put differently, with increasing degree of oxidation. It is, however, quite surprising that at low angle of incidence $\left(15^{\circ}\right)$ or normal incidence $\left(0^{\circ}\right)$, peaks II and III still show up, although the intensity is very low. In both cases, however, peak $I$ is not present, giving rise to an increase of band gap for these altered layers which are becoming more and more oxidelike structures.

It is important to note that at normal incidence (corresponding to $100 \%$ oxidation according to the Si $2 p$ photoelectron peak deconvolution) peaks II and $I I$ are still present. This can be explained as a result of ion-induced damage of the builtup $\mathrm{SiO}_{2}$ layer, resulting in an altered layer with some molecules formed by three oxygen atoms bonding to one $\mathrm{Si}$ atom. The fourth oxygen atom necessary to form the $\mathrm{SiO}_{2}$ molecule is sputtered by the ion beam leaving an unpaired $p$-like orbital (based on its binding energy) with similar characteristics as a Si $3 p$-like orbital peak. This peak is measured in region $I$ of the valence band but cannot be seen in the Si $2 p$ photoelectron region. Indeed the chemical environment of the $\mathrm{Si} 2 p$ core level in this molecule is comparable to a $\mathrm{SiO}_{2}$ environment, and therefore no chemically induced shift is present or it is too low to be measured with our energy resolution. If the unsaturated bond is located on the surface, it could react with water vapor to form a $\mathrm{Si}-\mathrm{OH} /$ $\mathrm{Si}-\mathrm{H}$ termination, which would give rise to a peak in the Si $2 p$ region too small to be detected.

The peaks corresponding to the nonbonding $\mathrm{O} 2 p$ orbitals (IV and $V$ ) remain, regardless of the angle of incidence, in a ratio of about $1: 1$ as in the $\mathrm{SiO}_{2}$ case. The energy position of these peaks is constant and in good agreement with the corresponding $\mathrm{SiO}_{2}$ peaks. For peaks VI and VIII we measure a very similar dependence with the angle of incidence, i.e., an increase in intensity with increasing degree of oxidation, which confirms the link between these peaks with the Si-O bonding orbitais. At $100 \%$ oxidation, peaks VI and VIII do not reach the $\mathrm{SiO}_{2}$ intensity, probably due to the induced damage as already pointed out for peaks II and III. The intermediate peak VII decreases with decreasing angle of incidence in contrast with the other bonding orbitals. This decrease must be seen as a lowering of the contribution of $\mathrm{Si}-\mathrm{Si} s-s$-like orbitals, but not as a lowering of the bonding orbitals ( $\mathrm{Si}-\mathrm{O})$. The contribution of this peak is still important at an angle of incidence of $35^{\circ}$.

The above-discussed results indicate that the valence band of the altered layer can be described by a mixture of $\mathrm{Si}$ and $\mathrm{SiO}_{2}$. The most surprising feature is the presence of occupied states in the region corresponding to peaks I, II, and III for angles smaller than $30^{\circ}$, where the Si $2 p$ spectrum indicates complete or partial oxidation but always without the presence of the pure Si chemical state. The results indicate that the density of states in this region is correlated with the angle of incidence, suggesting an effect of these states on the SIMS yield. The obvious framework to include this contribution is the resonant tunneling model. Before doing this, it is important to realize that presently the model was only formulated for metals where the Fermi level defines the energy region with occupied states. For a semiconductor like Si, or an insulator like $\mathrm{SiO}_{2}$, we must substitute the Fermi level by the top of the valence band and take into account the fact that only a limited number of occupied states are present in this energy region.

These assumptions, in the case of $\mathrm{SiO}_{2}$, imply that the first ionization energy for $\mathrm{Si}$ (I) in the free space needs to be referenced to the top of the valence band of thermal $\mathrm{SiO}_{2}$ [2.1 eV instead of $8.1 \mathrm{eV}$ that refers to the vacuum level, since the energy distance from the $\mathrm{SiO}_{2}$ top of the valence band to the vacuum level is $10.2 \mathrm{eV}$ (Ref. 26)] and that there will be no possibility for tunneling, since the first ionization level for Si (1) lies in the band gap and no occupied electron states are available at that level. However, in the case of crystalline Si and the altered layers (at all angles of incidence) the Si (I) level lies in the region formed by peaks $1, I$, and III of their respective valence band, in which case the tunneling effect is possible, since the density of states at that energy is not zero.

Combining the results for the XPS valence band, degree of oxidation derived from the Si $2 p$ core level, $^{41}$ and the observed variations in the cmission of $\mathrm{Si}^{+}$as a function of the angle of incidence during oxygen bombardment, ${ }^{42-44}$ we propose the following model:

(1) Al angles of incidence larger than $30^{\circ}-35^{\circ}$, the silicon is gradually converted to an oxide-rich layer with properties, however (as derived from the valence band), which are still crystalline-silicon-like. The secondary ion emission is then dominated by the increase in local Si-O bonds (local bond breaking).

(2) At angles smaller than $30^{\circ}-35^{\circ}$, the silicon surface becomes an oxidelike structure. The surfaces become nearly completely oxidized $(80 \%-100 \%)$ and very little variation in the number of Si-O bonds takes place. However, the increased oxidation gives rise to drastic changes in the valence band structure, leading to variations in the tunneling probability.

The increased ionization can be understood as being due to the large number of $\mathrm{Si}-\mathrm{O}$ bonds and a reduced tunneling probability in going to $0^{\circ}$ (due to changes in the valence band).

\section{CONCLUSIONS}

The different degree of oxidation in the surface of the altered layer gives rise to variations in the $\mathrm{Si}^{+}$ion emission during SIMS measurements. Two rather different regimes (angles of incidence larger or smaller than $30^{\circ}$ ) can be observed for the evolution of the $\mathrm{Si}^{+}$-ion emission as a function of the oxygen incorporation. In this work a detailed analysis and interpretation of the valence band region was made. A comparison with the experimentally measured and theoretically derived $\mathrm{Si}$ valence band and $\mathrm{SiO}_{2}$ valence band suggests that the "new" valence bands for the altered layers are formed by a combination of those two. This arises from the fact that $\mathrm{Si}-\mathrm{Si}$ bonds are present in the silicon suboxide molecules, and therefore the corresponding $3 p-3 p$ Si-like subband, which extends towards the silicon Fermi level, forms the top of the respective new valence bands. Small variations 
in intensity and energy position for this subband have dramatic implications on the intensity of the $\mathrm{Si}^{+}$-ion emission during sputtering in SIMS measurements.

A correlation between the valence band variations and the resonant tunneling model for secondary ion emission is proposed. A model combining chemically enhanced emission and resonant tunneling effects has been suggested to explain the variations in ion emission during oxygen bombardment for Si targets.

${ }^{1} \mathrm{~K}$. Wittmaack, in Proceedings of the International Workshop on Inelastic Ion-Surface Collisions, Bell Laboratories, New Jersey, July 1976.

${ }^{2}$ Y. Homma and K. Wittmaack, Appl. Phys. A 50, 417 (1990).

${ }^{3}$ M. L. Yu and N. D. Lang, Phys. Rev. Lett. 50, 127 (1983).

${ }^{4} \mathrm{~K}$. Franzreb, A. Wucher, and H. Oechsner, Phys. Rev. B 43, 14396 (1991).

${ }^{5} \mathrm{P}$. Williams, in Secondary Ion Mass Spectrometry, SIMS VII, edited by A. Benninghoven, C. A. Evans, K. D. Mckeggan, H. A. Storms, and H. W. Werner (Wiley, New York, 1990), p. 15.

${ }^{6}$ M. I. Yu and N. D. Lang, Nucl. Instrum. Methods B 14, 403 (1986).

${ }^{7}$ H. Oechsner and Z. Sroubek, Surf. Sci. 127, 10 (1983).

${ }^{8}$ M. L. Yu and K. Mann, Phys. Rev. Lett. 57, 1476 (1986).

${ }^{9}$ M. L. Yu, Nucl. Instrum. Methods B 15, 151 (1986).

10\%. Sroubek, in Secondary Ion Mass Spectrometry, SIMS VI, edited by A. Benninghoven, A. M. Huber, and H. W. Werner (Wiley, New York, 1988), p. 17.

${ }^{11}$ M. L. Yu, in Ref. 10, p. 41.

${ }^{12}$ C. D. Wagner, W. M. Riggs, L. E. Davis, J. F. Mulder, and G. I. Muilenberg, Handbook of X-Ray Photoelectron Spectroscopy (Perkin-Elmer, Eden Prairie, 1979).

${ }^{13}$ D. Briggs and M. P. Seah, Practical Surface Analysis by Auger and X-Ray Photoelectron Spectroscopy (Wilcy, Chichester, 1983).

${ }^{14}$ P. J. Gruntbaner, M. H. Hecht, F. J. Grunthaner, and N. M. Johnson, J. Appl. Phys. 61, 629 (1987).

${ }^{15} \mathrm{C}$. Coudray and G. Slodzian, in Ref. 10 , p. 45.

${ }^{16}$ M. L. Cohen and J. R. Chelikowsky, in Handbook on Semiconductors, edited by T. S. Moss (North-Holland, Amsterdam, 1982) Chap. 4b, p. 219; Electronic Structure and Optical Properties of Semiconductors,
Springer Series in Solid-State Sciences No. 75, 2nd ed. (Springer, Berlin, 1989).

${ }^{17}$ L. Ley, S. Kowalczyk, R. Poilak, and D. A. Shirley, Phys. Rev. Lett. 29, 1088 (1972).

${ }^{18}$ M. L. Cohen and J. D. Joannopoulos (private communication).

${ }^{19}$ D. J. Stukel, T. C. Collins, and R. N. Euwema, in Electronic Density of States, Proceedings of the Third International Materials Research Symposium, Gaithersburg, Maryland, 1969, edited by 1. H. Bennett, National Bureau of Standards Special Publication No. 323 (U.S. GPO, Washington, DC, 1971).

${ }^{20}$ R. A. Pollak, L. Ley, S. Kowalczyk, and D. A. Shirley, Phys. Rev. Lett. 29, $1103(1972)$

${ }^{21}$ D. E. Eastman, W. D. Grobman, J. L. Freeouf, and M. Erbudak, Phys. Rev. B 9, 3473 (1974).

${ }^{22}$ G. Hollinger and F. J. Himpsel, J. Vac. Sci. Technol. A 1, 640 (1983).

${ }^{23}$ J. Tersoff and D. R. Hamann, Phys. Rev. B 28, 1168 (1983).

${ }^{24}$ J. Robertson, I. Phys. C 18, 947 (1985).

${ }^{25}$ P. S. Ho and G. W. Rubloff, Thin Solid Films 89, 433 (1982).

${ }^{26} \mathrm{H}$. Ibach and J. E. Rowe, Phys. Rev. B 10, 710 (1974).

${ }^{27}$ G. W. Rubloff, Phys. Rev. B 25, 4307 (1982).

${ }^{28}$ B. Fischer, R. A. Pollak, T. H. DiStefano, and W. D. Grobman, Phys. Rev. B 15, 3193 (1977).

${ }^{29}$ T. H. DiStefano and D. E. Fastman, Phys. Rev. Lett. 27, 1560 (1971).

${ }^{30}$ S. Ciraci and S. Ellialtioglu, Solid State Commun. 40, 587 (1981).

${ }^{31}$ A. Balcarotti and A. Bianconi, Phys. Status Solidi B 76, 689 (1976).

${ }^{32}$ R. B. Laughlin and J. D. Joannopoulos, Phys. Rev. B 21, 5733 (1980).

${ }^{33}$ M. H. Reilly, J. Phys. Chem. Solids 31, 1041 (1970).

${ }^{34}$ S. Ciraci and I. P. Batra, Phys. Rev. B 28, 982 (1983).

${ }^{35}$ R. B. Laughlin, Phys. Rev. B 22, 3031 (1980).

${ }^{35}$ S. P. Kowalcayk, F. R. McFeely, L. Ley, V. T. Gritsyna, and D. A. Shirley, Solid Staie Commun. 23, 161 (1977).

${ }^{37}$ V. M. Bermudez and V. Ritz, Phys. Rev. B 20, 3446 (1979).

${ }^{38}$ J. Pollmann and S. T. Pantelides, Phys. Rev. B 18, 5524 (1978).

${ }^{39}$ J. A. Tossell, D. J. Vaughan, and K. H. Johnson, Chem. Phys. Lett. 20, 329 (1973).

40 J. A. Tossell, J. Am. Chem. Soc. 97, 4840 (1975).

${ }^{41}$ J. L. Alay and W. Vandervorst, Surf. Interface Anal. 19, 313 (1992).

${ }^{42}$ Y. Homma and K. Wittmaack, J. Appl. Phys. 65, 5061 (1989).

${ }^{43} \mathrm{~K}$. Wittmaack, Nucl. Instrum. Methods 218, 307 (1983).

${ }^{44} \mathrm{~K}$. Witmaack, Nucl. Instrum. Methods B 2, 674 (1984). 